\title{
Virtual Training for Industrial Process: Pumping System
}

\author{
Edison P. Yugcha, Jonathan I. Ubilluz, and Víctor H. Andaluz
}

Universidad de las Fuerzas Armadas ESPE, Sangolquí-Ecuador

\{epyugcha,jiubilluz, vhandaluzl\} @espe.edu.ec

\begin{abstract}
The article presents a virtual environment of a pumping system oriented to training of users that interact with industrial processes. The application of it was performed in a graphic engine Unity $3 \mathrm{D}$, where shows two training environments: i) Electro pumps laboratory, simulates control operations of control for manipulating many configurations from centrifugal pumps in individual, serie or parallel in order to visualize by an HMI the physical parameters such as: pressure, flow and temperature; ii) Industrial environment the user prepares in a complementary way how to know industrial processes in a practical and realistic way. In order for making the virtual application immersive and interactive, the modeling of the electrical characteristics of the pumping system was carried out.
\end{abstract}

Keywords: Virtual Environment, Pumped System, Unity 3D, Immersive and Interactive.

\section{$1 \quad$ Introduction}

Nowadays, the virtual technology has been implemented in several environments such as: education, medicine and especially at industries, this one allows to develop the catchment level and with it the understanding of the work environment that all staff in the industry must have [1]. The industrial processes always require to get support, calibration and adjustments of each system's instrument, where a lot of them are dangerous to the operator who doesn't have enough training [2], in order to avoid that the company has required to different alternatives where the process does not represent any danger for the operator and machine, e.g., industrial digitalization where the operator can visualize the behavior of the machine. The use of virtual entertainment results a better experience for the operator, duo to the fact that it can interact with elements of the system and at the same time to watch all different changes [3]. By opting virtual technology for information and management of industrial equipment, it presents better results and reduces costs compared to the use of usual technologies [4].

At present induction videos and virtual tours are used by the industry for training all the staff, which seems to be very theoretical because It does not do any interaction with the other industrial processes, to satisfy the demands, RV and AR technologies are being developed, those ones allow to improve the training scenarios [5]. Some applications stand out in the field of design optimization, maintenance, process control and operator training e.g., there is a virtual application with focuses on the operator training, to simulate experiences in the decision-making that takes place in the field work [6], 
only focuses on operator training and not on the interaction with the process. The RV allows the user to interact with elements of the virtual world with a realistic approach, in this sense it can perform training scenarios for the operator's simulation under specific conditions [7]. On the other hand, the AR shows in the real world fictitious elements generated in a virtual way, which one can be used through software [8].

Petroleum processes tend to work continuously, with periodic maintenance planning and no work stoppages, duo to that it has led to staff training through virtual scenarios that sever to evaluate possible cases that can happen with the real equipment, by applying the RV it is not necessary the presence of the operator in the industry [9]. One if the methods of virtual in the pumping system is the extraction of crude oil by means of centrifugal pumps from the production wells and displacing them by pipes with the objective of the discharge is at ground level [10]. Besides there's an area of industrial maintenance where the operator can manipulate the pitching and receiving traps of the pipe scrapers (PIG) in order to strengthen knowledge [11]. The detection of virtual modules in existing plant topologies by means of extended search algorithms and the definition of functional patterns, it is now possible to identify all virtual modules within a plant topology [12]. By the necessity of modernizing the industry's facilities digital models are implemented for simulation in a virtualized environment, which allows to automatically convert engineering documents obtained specifically from pipe diagrams and instrumentation. The resulting models can serve as a basis to support engineering tasks that require simulations [13]. The applications that were mentioned take focus on the training of professionals but not the educational field.

This work presents a virtual application aimed at training users in the pumping system, in order to solve this trouble, it has been developed two scenes that allow immersion and interaction through the graphic engine Unity 3D and through mathematical models to do the system analysis. i) Electro pump laboratory has disposed of several pumping system modules where the user can simulate control operations, supervision and data acquisition; how opening or closing of valves to configure VFD parameters, perform configurations of centrifugal pumps in individual, serie or parallel, and visualize temperature data in a graphical interface (HMI) that are obtained by modeling the electrical characteristics, while in the ii) Industrial environment, allows to know industrial processes in a practical and realistic way, so that the user is trained to perform maintenance practices through didactic instructions, in this method it is able to see the risks that can occur when an incorrect maneuver is performed. Finally, to obtain a more realistic application is implemented a safety signage, surround sounds and the use of external devices to interact with the environment.

The article is structured in 6 sections including the Introduction. Section 2 describes the development of the virtual application using blocks; In Section 3 it is represented by stages for the development of the virtual environment. Section 4 shows the behavior of the system. Section 5 shows the results obtained in the investigation; to finally, establish the conclusions in Section 6. 


\section{Problem Formulation}

For the development of the virtual environment of a pumping system, scripts are used, those ones are the principal part of the application because they allow to link different stages such as the input and output devices, through the use of virtual reality glasses and optical controls, the mathematical model of the system is performed in the Matlab Software and by using shared memories it's linked to Unity 3D software for sending and receiving data, with it the user will be able to interact with different work scenes that contain the virtual environment to establish a greater realism in the interaction and immersion between the user and the 3D virtual pumping system.

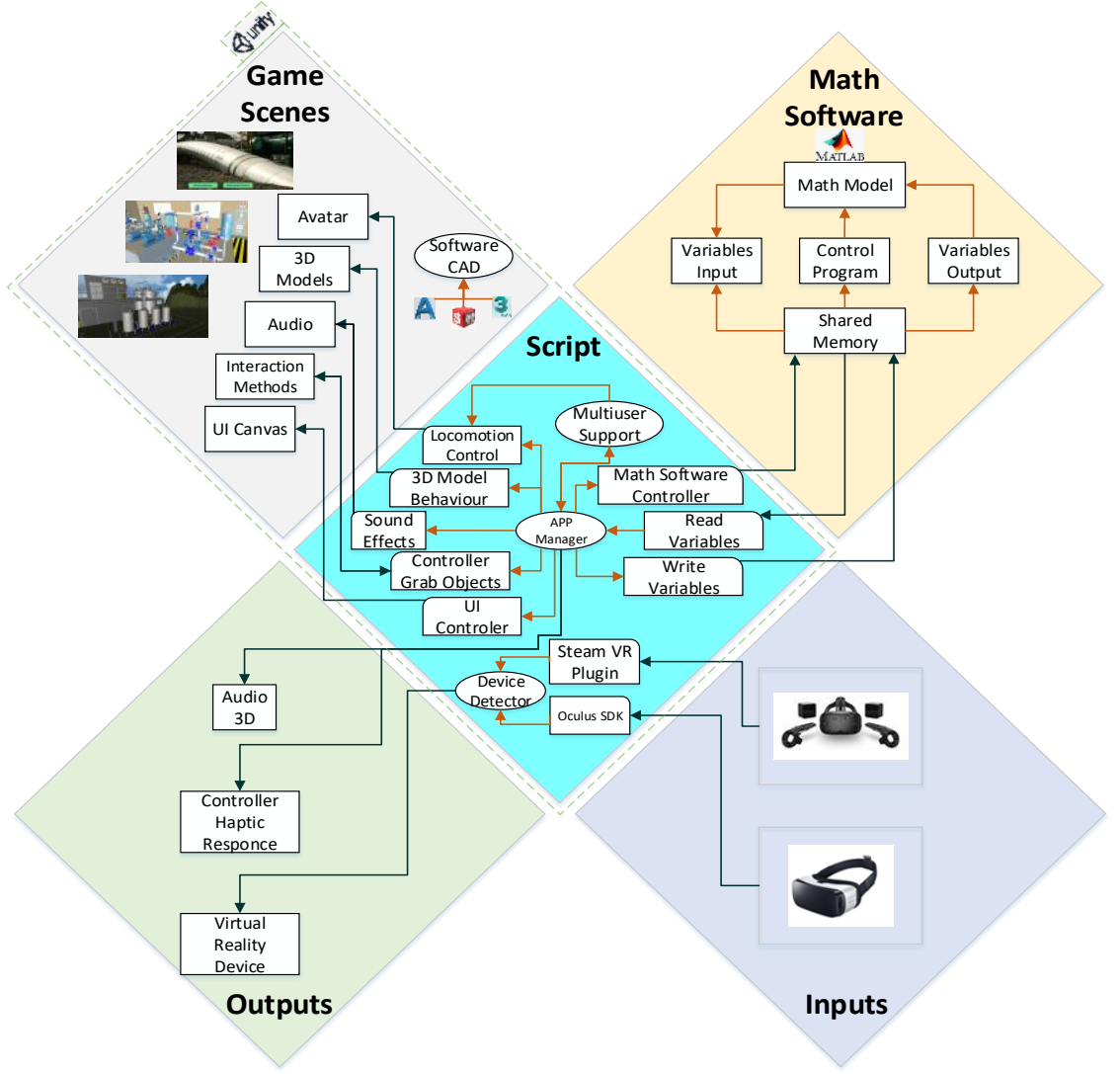

Figure 1. Block diagram of interrelation between components

Figure 1. Shows different blocks for application's development. The first section called SCENES, represents the content of GAME OBJECTS for the edition of pumping system variables. In this phase the interaction between the user and the application is configurated by the Controller Grab Objects which allows to take over and supervise the virtual environment behavior. 
The Scripts block indicates the communication between the Unity 3D AppManager, mathematical software and the input and output devices. Through a set of GUI extensions allow the game developer to insert windows, panels, buttons among others to visualize the behavior of electrical variables, when interacting with the user.

The mathematical software covers the mathematical modeling of the electrical characteristics of the pumping system, for which input variables of the frequency inverter (speed) and the type of configuration of the hydraulic circuit are used by the centrifugal pumps that are controlled through a HMI, where the user can monitor the process, to obtain the physical variables of output such as flow, pressure and temperature. Finally, the connection between Unity 3D and Matlab is made through shared memories that allow sending and receiving data.

In the entry stage, two devices are considered: i) Oculus SDK, allows the user to immerse for the control of movements and the angle of vision. ii) Controller Grab $\mathrm{Ob}$ ject, is responsible for the control of user functions, while in the output stage the sounds that are generated in the virtual environment are emulated when the pumping system process starts, it also shows the output variables through an HMI implemented in the virtual laboratory, so that the user has a more realistic sensation.

\section{Development}

In Figure 2, it's shown the pumping process in order to be virtualized, for which the instrumentation and pipe diagrams (P\&ID) are used, which allow maintenance and modification of the process [14]. The P\&ID diagram is developed in the AutoCAD P\&ID software that contains 2D CAD designs of mechanical and electronic components such as: centrifugal pumps, tank and measuring equipment that emulate the operation of the pumping system.

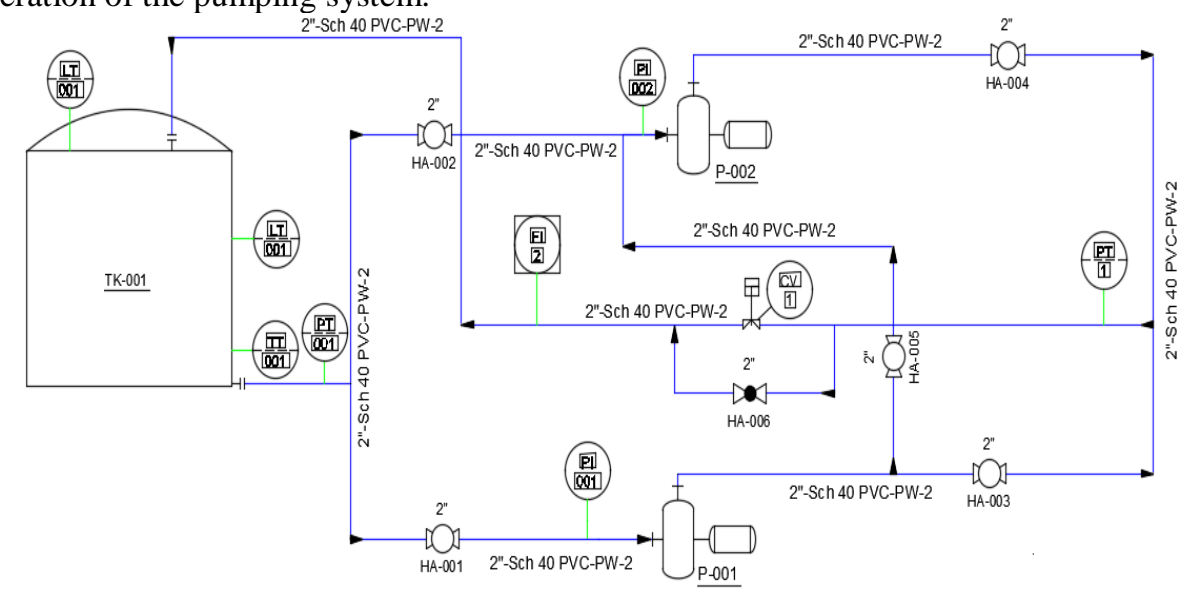

Figure 2. P\&ID Diagram of pumping system

In Figure 3, the different stages for the digitization of tools and instrumentation equipment are described, those one will be visualized in the virtual environment. 


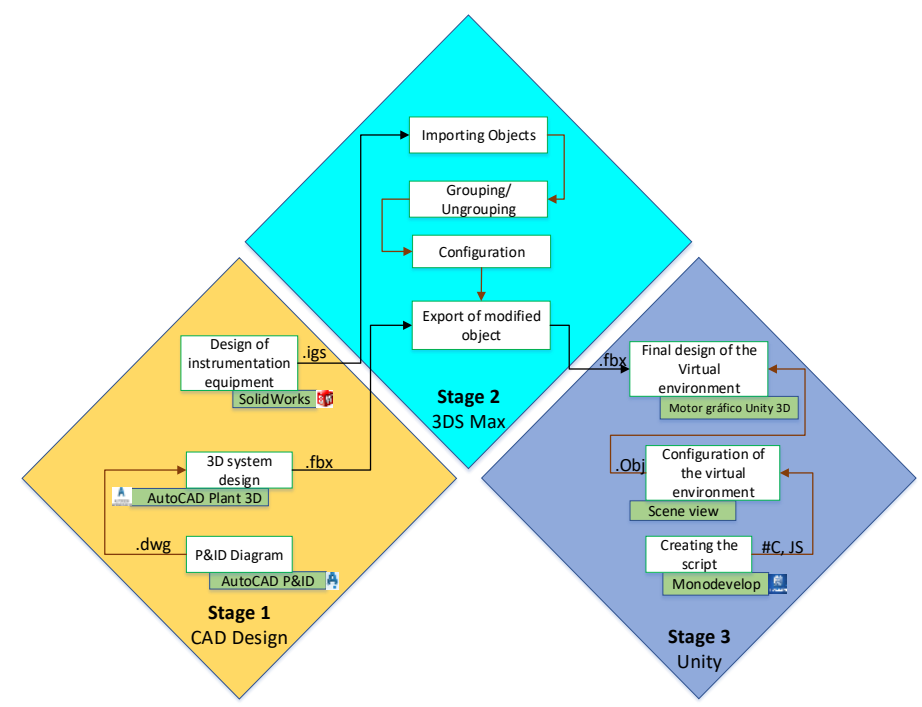

Figure 3. Virtualization stages

Stage 1. In the CAD design phase, AutoCad Plant 3D software is used to design and model piping systems for industrial processes, the advantage is that components to be implemented are governed by catalog standards. The design of all components of the pumping system must be exported with the extension *. FBX, e.g., centrifugal pumps, valves, pipes, among others, as shown in Figure 4.

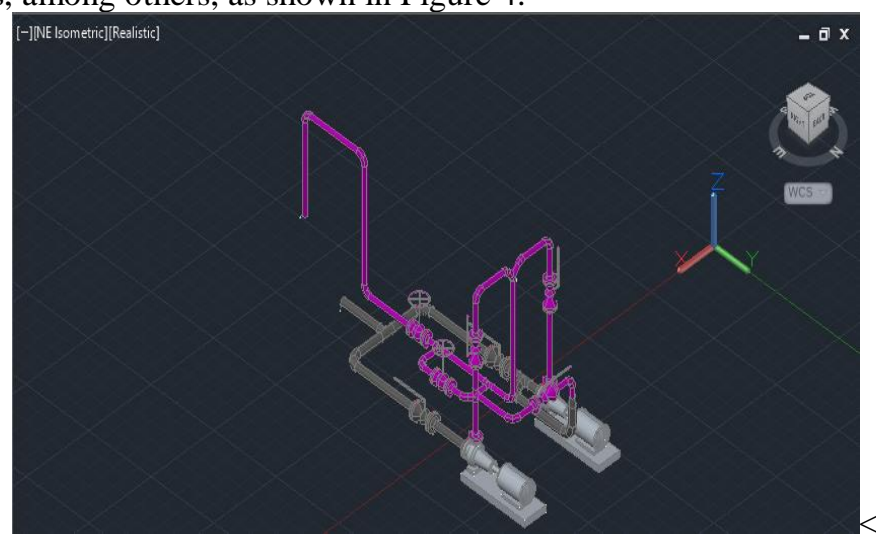

Figure 4. 3D Pumping system design

Stage 2. In this stage we used SolidWorks software that allows the creation and assembly of 3D elements, these designs must be exported with the extension. IGS because it's compatible with the 3DS MAX software, this one allows to group or uncouple the parts of the model in groups without incurring in the relationships of position and movement created, finally to export the created file to UNITY 3D with extension FBX, e.g. Flow meter, flow sensor, pressure, instrumentation components, laboratory accessories, 
and others. Figure 5, shows the comparison between the virtualized instruments with respect to the real instruments.

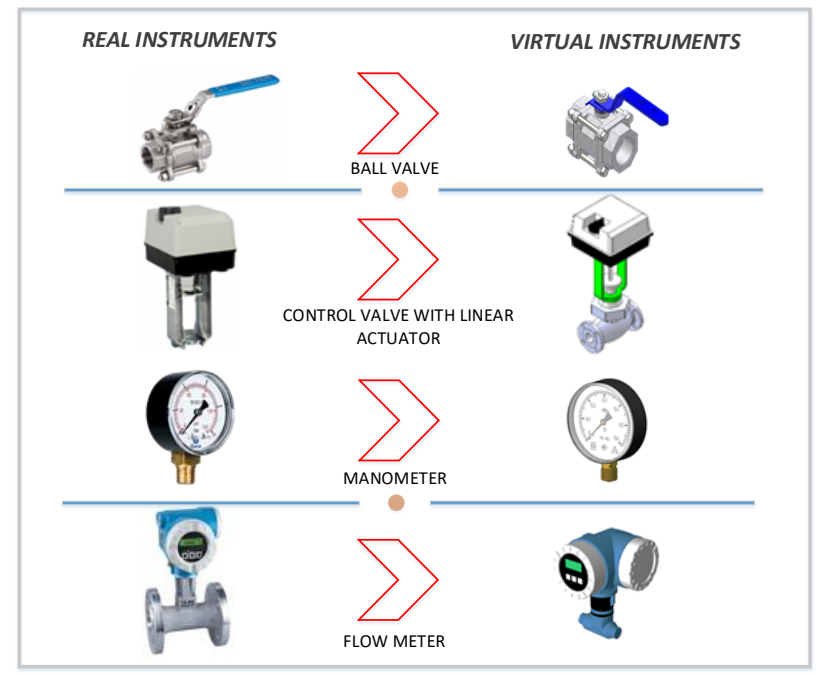

Figure 5. Shows the comparison between virtualized instruments with respect to real instruments.

Stage 3. In the third stage, the virtual environment is developed using the Unity 3D Graphic Engine, where the virtual simulation is carried out through different scenes, which show the training modes. The configuration of the HTC VIVE and Gear VR devices allow a better interaction between the user to explore and interact with the pumping system, by a programming in scripts such as e.g., i) Physical Events On Collision (Enter, Stay, Exit) and On Trigger (Enter, Stay, Exit); events that occur when colliding and physically interacting with the object. ii) The event controller for the user to interact with the environment is imported Package of Steam VR Plugin provided by the Asset Store. The user can move around the virtual environment using the HTC teleportation system. In addition, in the main window of Unity is located the Asset in which folders are created for animations, scripts, textures and materials to organize each event of the pumping system, see Figure 6.

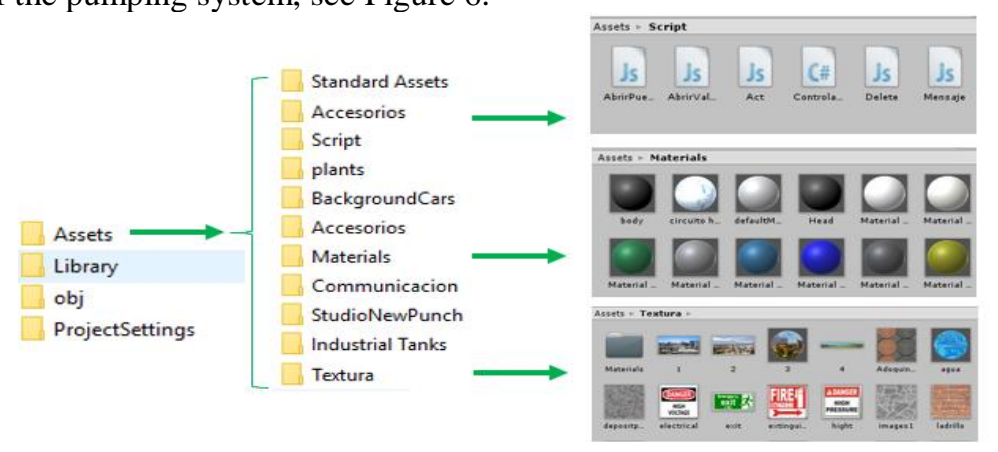

Figure 6. Distribution of parameters for the development of the virtual environment 
By giving more realism to the virtual environment textures and physical properties are added, also, scripts are used for the animation of the process to be carried out e.g., the opening and closing of the control valve with linear actuator, as indicated in Figure 7.

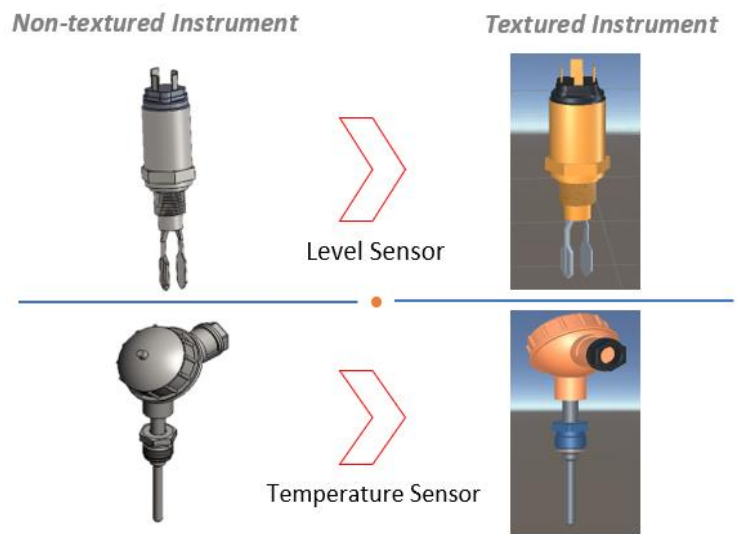

a) Characteristics of textures in virtual instruments

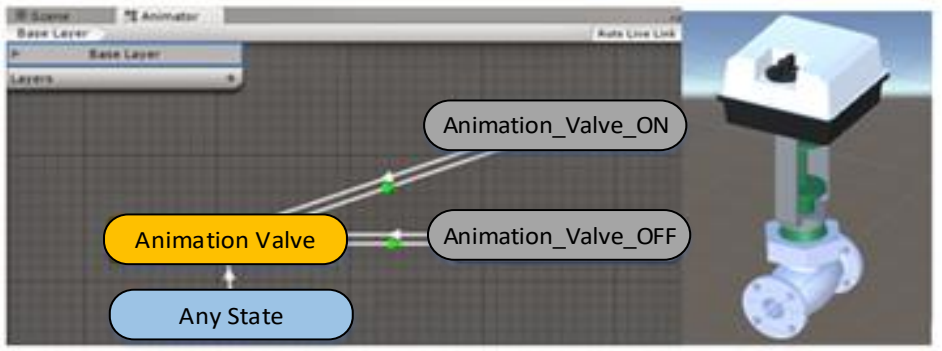

b) Animation of instruments

Figure 7. Instruments in the Unity 3D editor

\section{System Behavior}

Because of the virtual environment has similarity with reality, the data of the virtual laboratory are linked with the data generated in the mathematical processing, where the variables and operating conditions that involve the pumping system are specified, at this it will be obtained an answer in real time to the variable modifications that made some user.

The interchange of input and output data is done in a distributed way through different partitions in a shared memory, duo to the fact that they allow to process more information in an orderly and dynamic between the software Matlab and Unity 3D. In Figure 8. The bidirectional interaction between the pressure $(P)$, flow $(Q)$, temperature $(T)$ and valve position $(O N / O F F)$ data is indicated, in order to emulate the behavior of the 
plant through mathematical processing which varies according to the actions performed by the user in the virtual environment.

Position valve (ON/OFF)

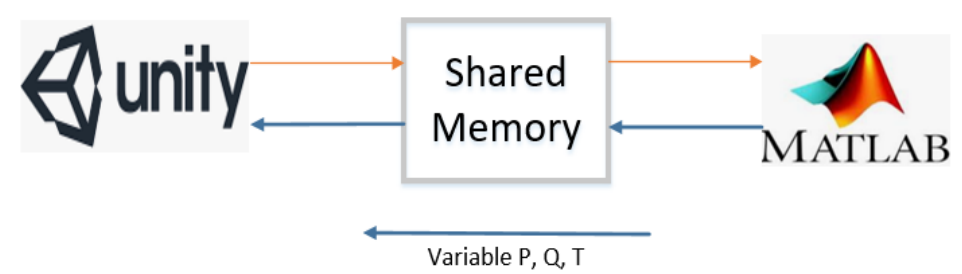

Figure 8. Data interaction between Unity 3D Software and Matlab

Mathematical modeling is important because it allows to simulate the interaction between the pumping system and the user. This is done using MathWorks Simulink software that offers a wide variety of mathematical engineering elements, in this case the modeling of a centrifugal pump and valves was obtained, as shown in Figure 9. In the Matlab - Simulink software [15]., the frequency inverter (VFD) is programmed to modify the electrical parameters of the centrifugal pump such as frequency, nominal speed, power, current, and in the same way the configuration of the valves in individual, serie and parallel.

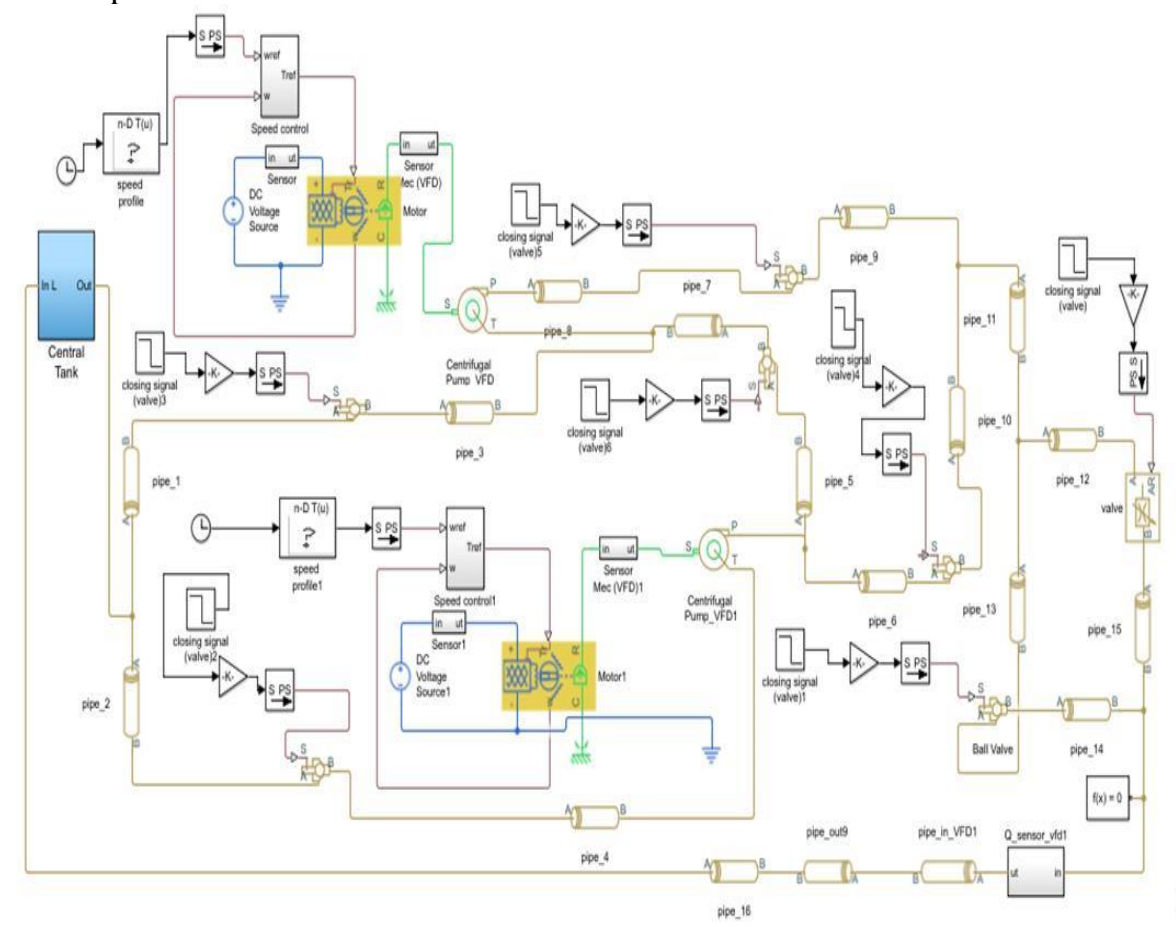

Figure 9. Mathematical model of the pumping system 


\section{$5 \quad$ Results and Discussion}

In this section, the results reached in the virtual application developed are shown, as a novel alternative for the training of users in industrial processes, because real laboratories do not emulate large processes due to lack of equipment, instruments, budget or infrastructure. The virtual training module in the area of Pumping System allows the user to know in a practical way the industrial processes and the consequences when performing incorrect maneuvers.

The virtual environment shows two training modes that allow user interaction and immersion and can be selected according to the need for learning, as shown in Figure 10 .

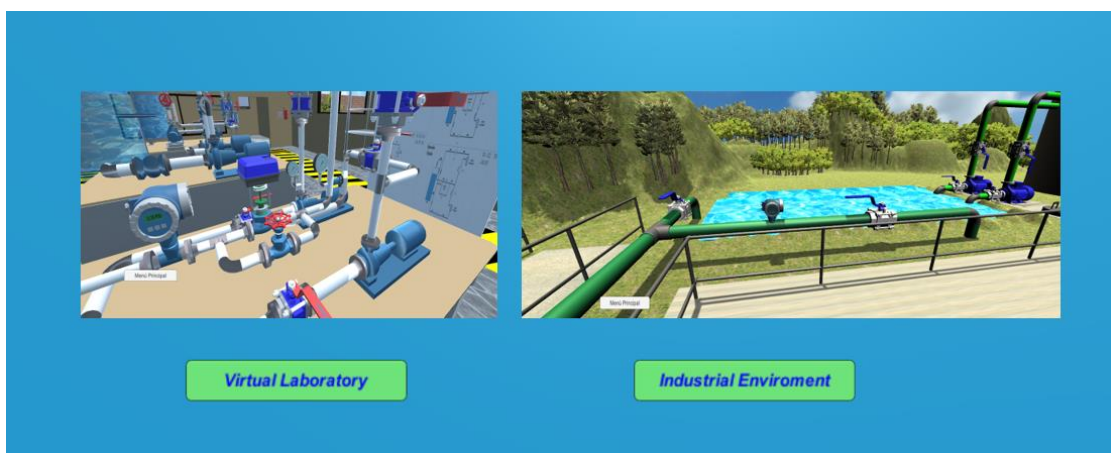

Figure 10. Scene selection

Virtual Laboratory Mode, in order to change the conventional teaching methodology, additional operating instructions are used in the form of dialog boxes that the user can observe during the development of the laboratory practice in the area of pumping system. In addition, the laboratory is equipped with electrical and mechanical tools such as, e, g., Screwdrivers, wrenches, clamps, centrifugal pumps, frequency inverter, valves and measuring equipment that allows to emulate maintenance tasks. In this training mode the user can assemble pumping systems according to the configuration of the centrifugal pumps, i.e., individual, serie or parallel, see Figure 11.

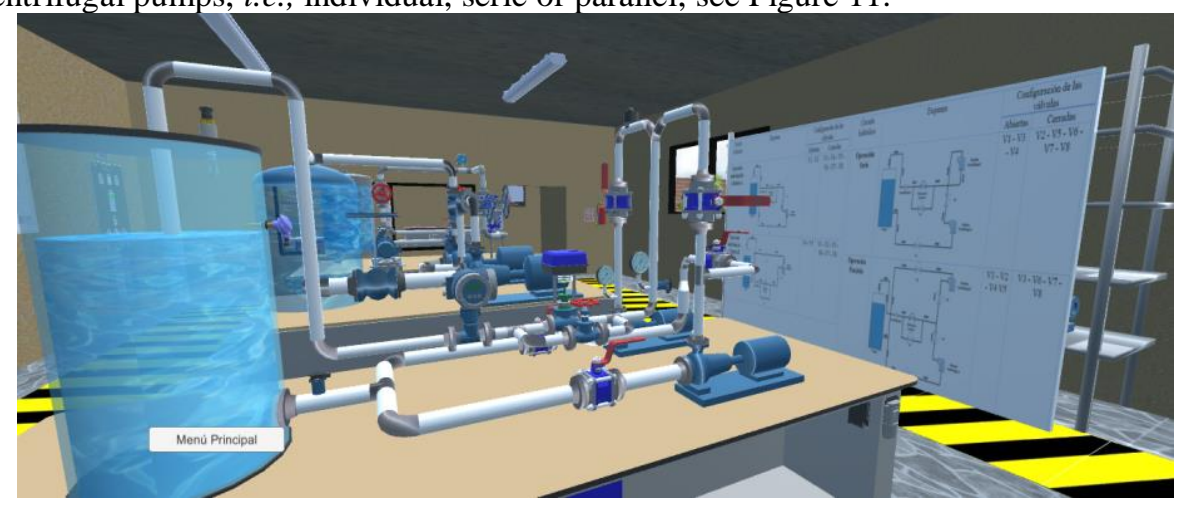

Figure 11. Virtual lab 
The virtual application data is obtained from the real pumping system as shown in Figure 12, through the LocalHost base that receives the data from the PLC, with the use of Xampp software real process information is stored in LocalHost and through MySQL manage the data in Unity $3 \mathrm{D}$, with a unidirectional communication.

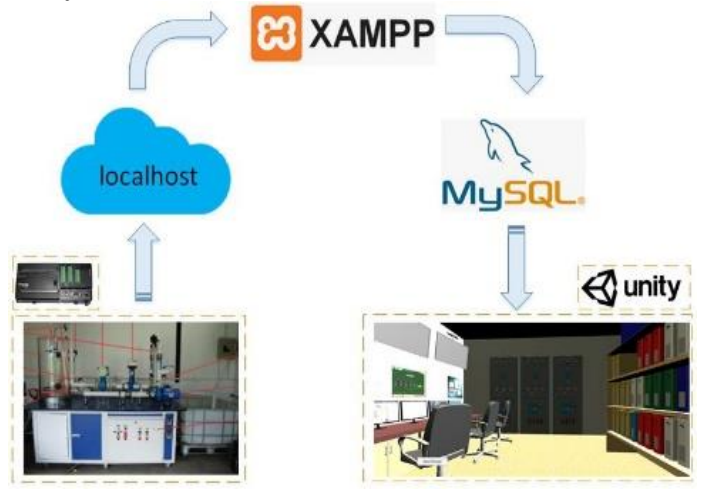

Figure12. Communication between the physical system and the virtual environment

To validate the mathematical model, an experimental comparison test was used between the efficiency curves generated in the Matlab graphical editor and the LocalHost database with data generated by the actual pumping system. In Figure 13, the curves are shown in a) Individual, b) Serie or c) Parallel, the serie configuration maintains the flow while the parallel configuration increases due to the change in the suction and discharge pressure of each pump.

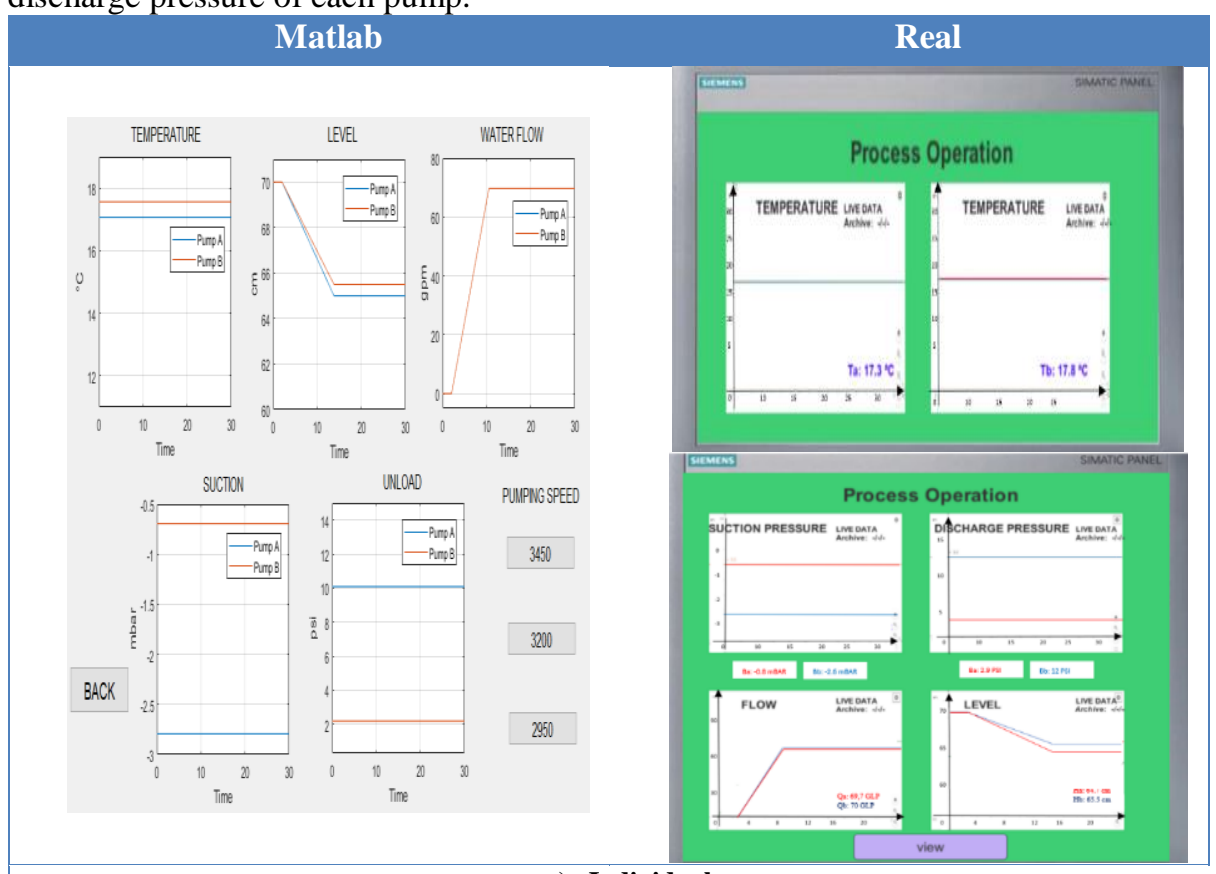

a) Individual 


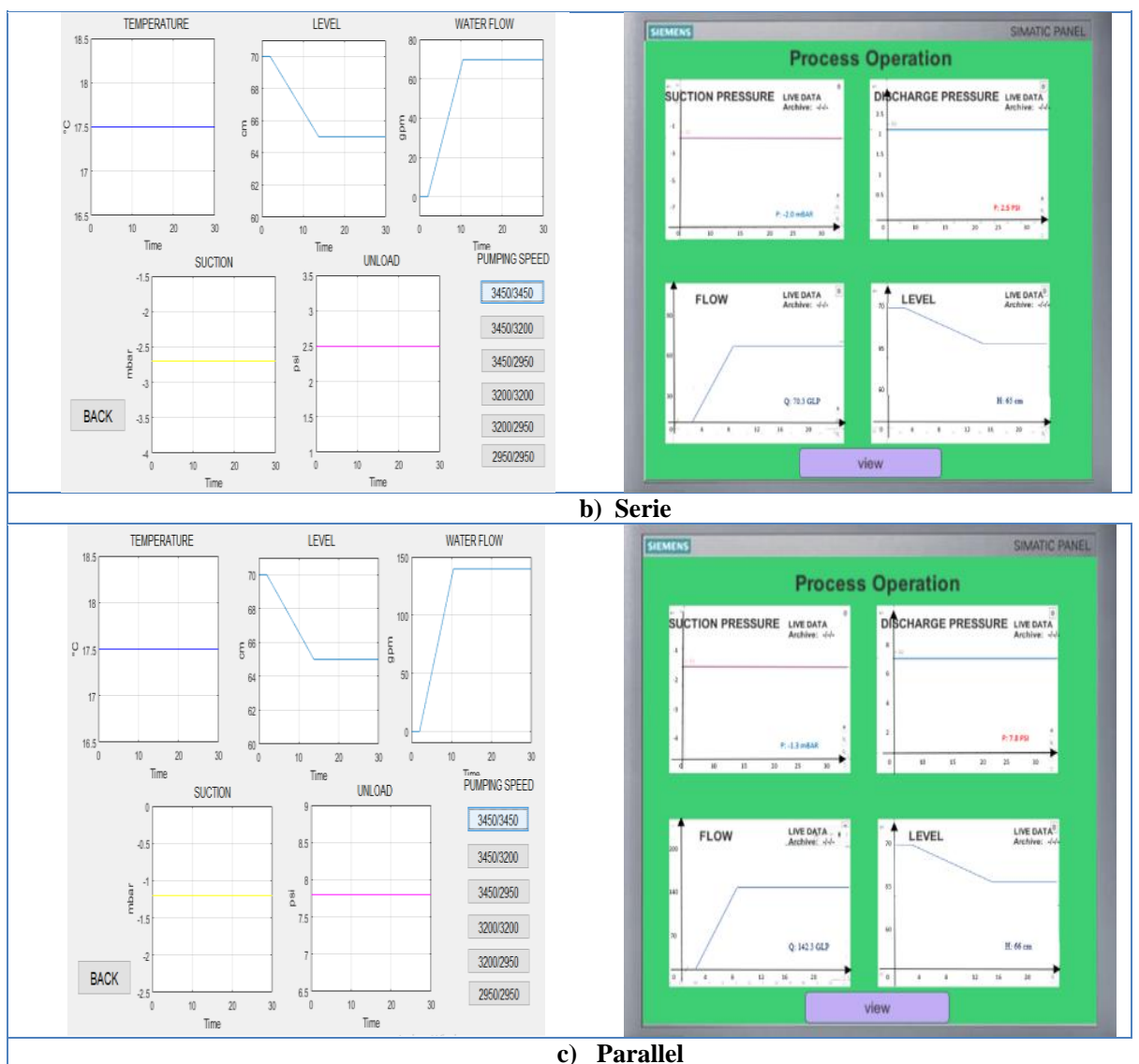

Figure 13. Efficiency curves of the virtual system

The curves generated in the real system and in the Matlab software, have similarity what allows to verify that the mathematical model applied in the virtual application is correct, and allows to interpret the operation of the real system.

Industrial Environment Mode, the route in the environment does not replace the physical training, but it allows to know maintenance tasks that it doesn't have a planning of the industry, it is difficult to have access. The virtual application presents a great advantage for the training of students and new workers who do not have knowledge in real industrial processes, with the implantation of the virtual industrial environment, the probability of learning in the area of the pumping system increases. As shown in Figure 14. 


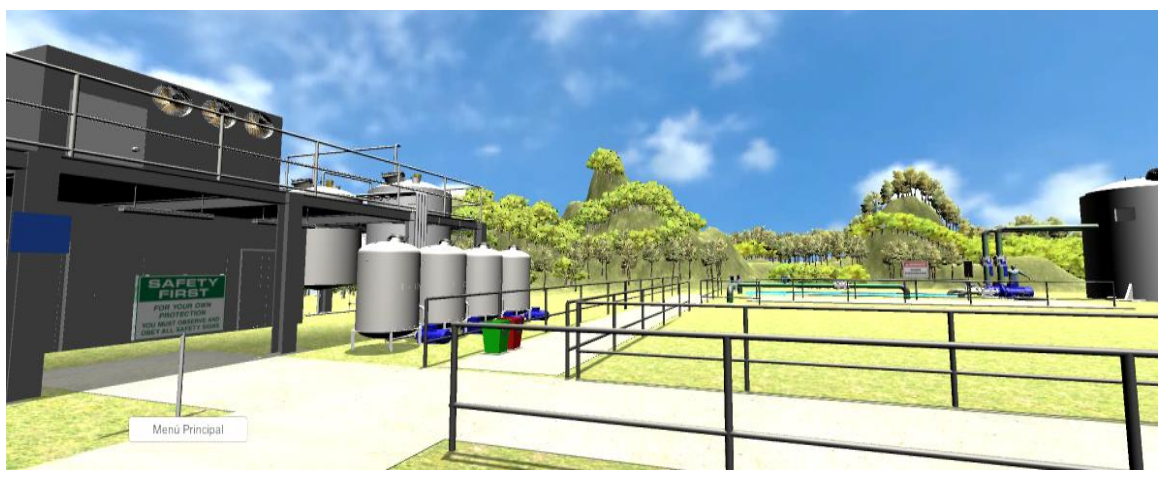

Figure 14. Industrial environment

In order to avoid that users, suffer any accident both the virtual laboratory and the industrial environment, operational, safety and prevention information is implemented according to the INEN-ISO 3864-1 standard [16], this one allows to avoid situations of risk in any training mode, they are strategically located in different places with the objective that any user can easily detect them [17], as shown in Figure 15 and 16.

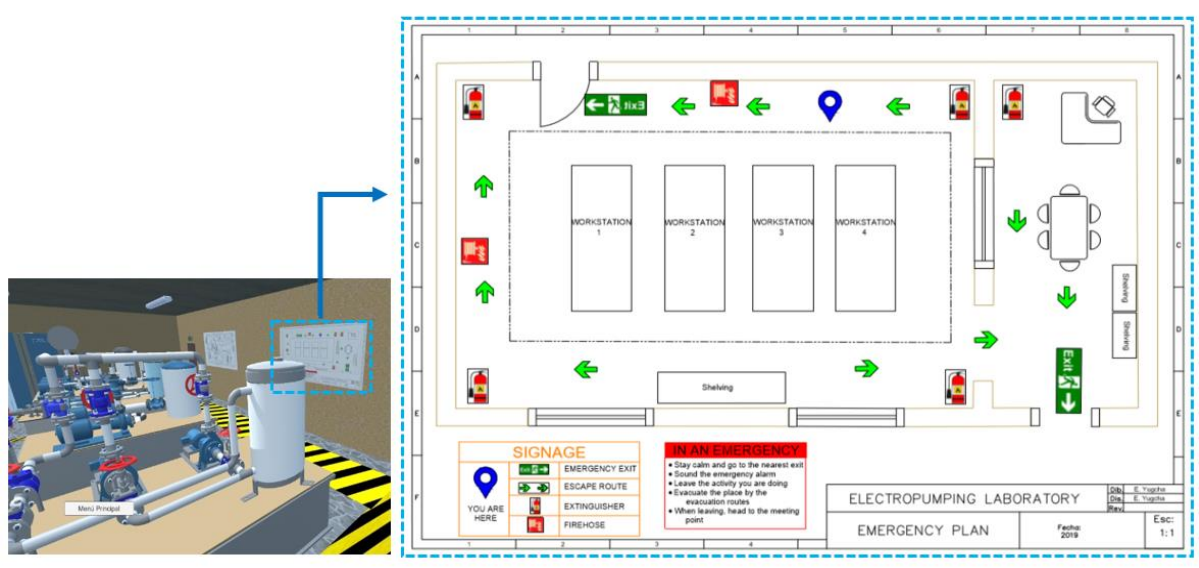

Figure 15. Evacuation plan in the laboratory
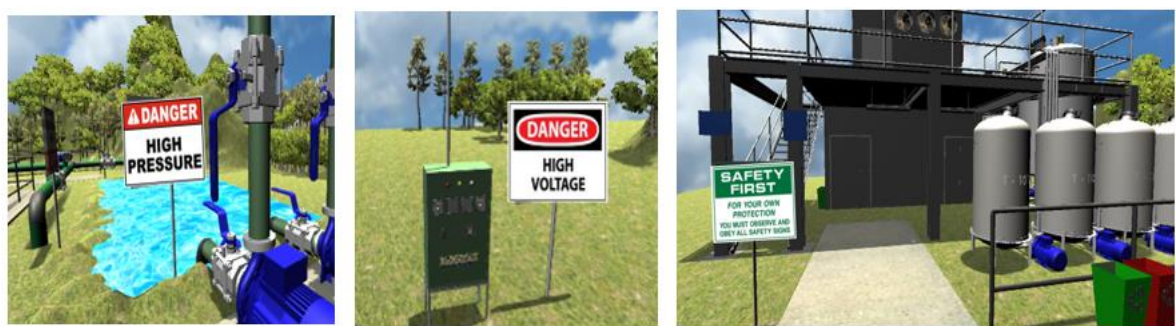

Figure 16. Safety signs in the industrial environment 
In the training modes situations of risks are development that are activated when the user does not comply with the operating protocols, in this way, more realism is given to the virtual environment. An emergency situation occurs when the user causes accidental changes to the work plan or to perform different instructions in the process $e . g$., i) Serial configuration: valve 3 is closed and causes a pressure rise in the suction pipe, ii) Parallel configuration: raises the excess pressure in the suction and discharge pipes when valves 3 and 4 are closed. In both situations, if the problem is not controlled, it can cause the pipes to explode, mechanical and electrical faults in the centrifugal pump. These emergency situations are visualized when the pipe changes to a red color, see Figure 17 and 18.

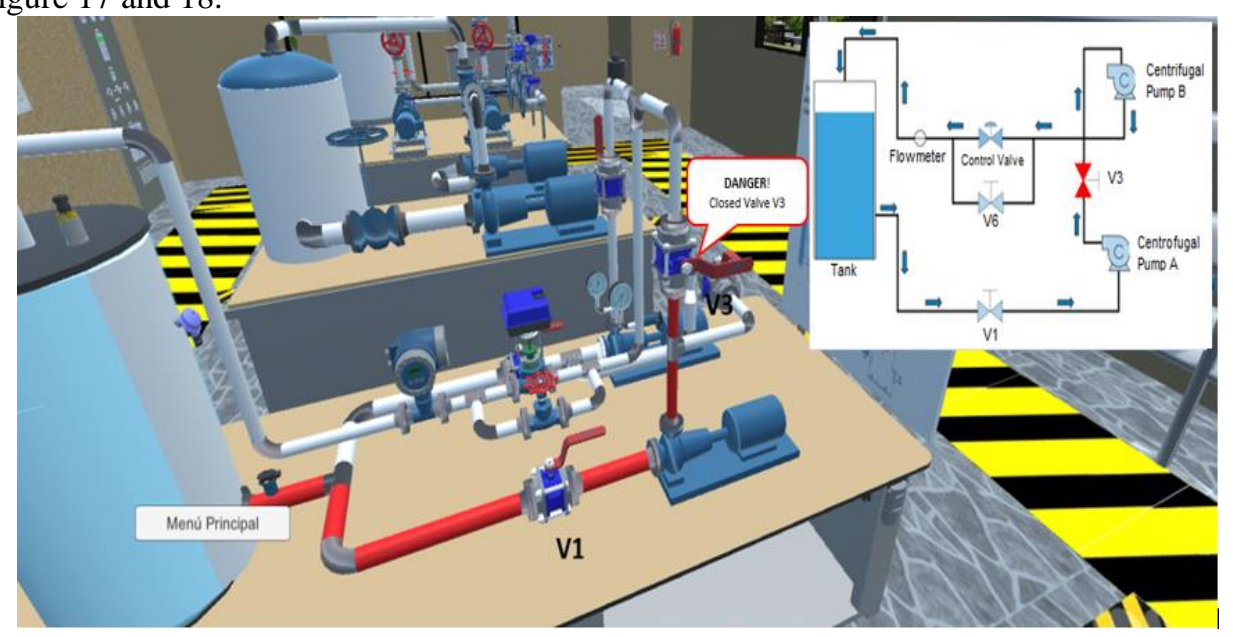

Figure 17. Emergency situation in serial configuration

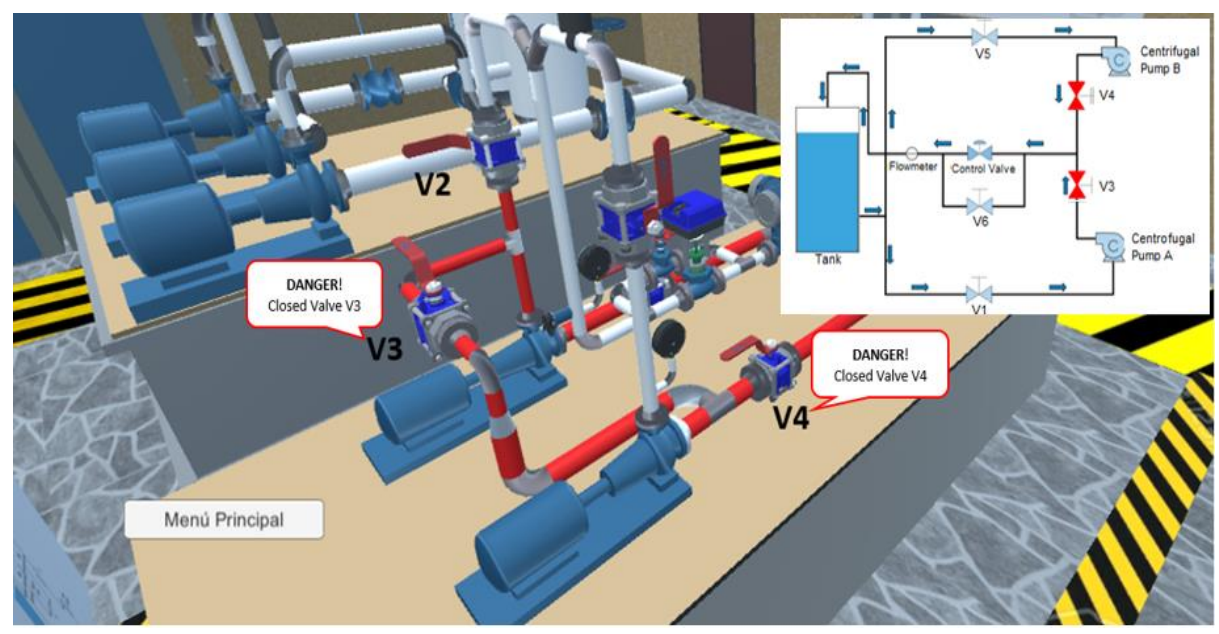

Figure 18. Emergency situation in parallel configuration 
The application of this one presents the training modes with the inclusion of an avatar that simulates an assistant who is determined to make instruction to the users in specific places into the virtual mode. With this a better immersion is obtained, in order that the user can take guided tours for leaning or training in the area of pumping system, as indicated in Figure 19.

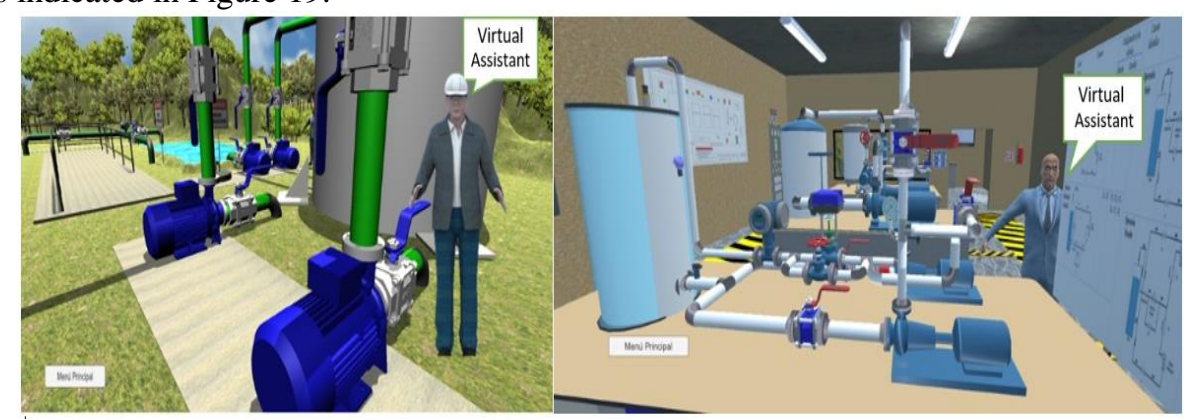

Figure 19. Virtual assistant

To determine the efficiency of the virtual application, usability test was used to different users in chairs e.g., Fluid Mechanics, Plant Design, Industrial Instrumentation and Process Control to determine the experience in the virtual training environment, as indicated in Table 1.

Table 1. The usability of the virtual application

\section{QUESTIONS}

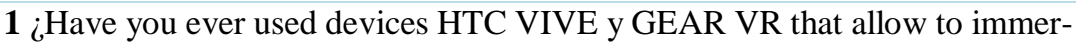
sion and interact in virtual environments?

$2 i$ The use of the training module is intuitive?

3 . Does it result easy the management of devices in the virtual environment? $4 i$ Are you able to perform the operation given by the virtual assistant?

5 ¿Could you identify risk conditions and emergency situations in a real industrial environment?

6 ¿Are the operating modes in the Pump System clear?

7 Would you recommend the training module as an additional tool to the theoretical training that is given in the educational and industrial field?

8 ¿Did the virtual application help you understand and improve skills in industrial processes?

9 ¿Do the safety signals placed in the virtual environment comply with the established standards?

$\mathbf{1 0}$ ¿Does the incorporation of new technologies (RV) allow the development of practices with problems of real industrial processes?

Figure 20, indicates the standard deviation of the users surveyed for each question, these results show that the virtual application is accepted by users as a training module 
in the Pumping System area, where the achievement of the application is evaluated between the range of regular (1) and excellent (5).

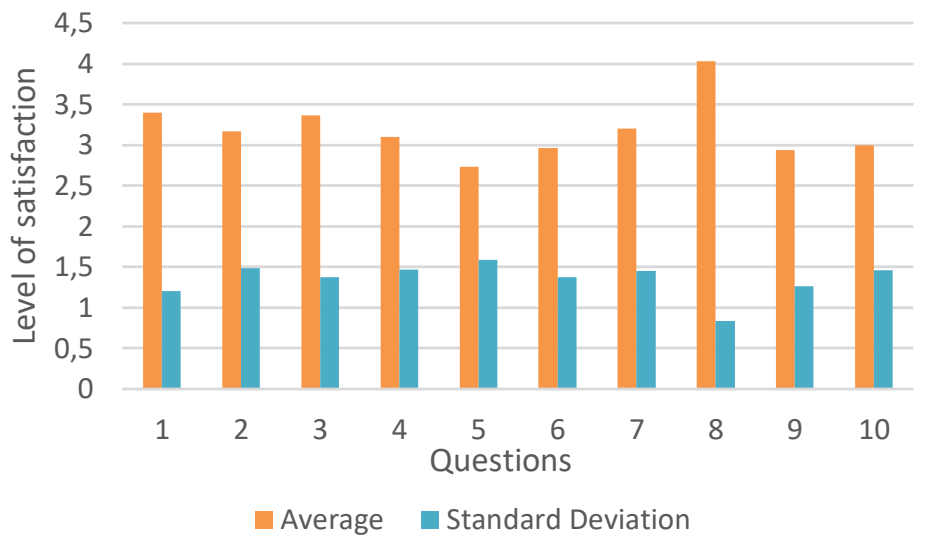

Figure 20. User learning level within the virtual environment

\section{Conclusions}

The article presents the development of a virtual application containing pumping plants and 3D measurement and control instruments designed in the software AutoCad Plant 3D and SolidWorks respectively, the files are exported to the Unity 3D graphics engine to give realism to the working environment and through manipulation devices such as HTC VIVE and Gear VR allow the user to immerse and interact realistic with the pumping process.

Acknowledgements. The authors would like to thanks to the Corporación Ecuatoriana para el Desarrollo de la Investigación y Academia-CEDIA for the financing given to research, development, and innovation, through the CEPRA projects, especially the project CEPRA-XI-2017-06; Control Coordinado Multi-operador aplicado a un robot Manipulador Aéreo; also to Universidad de las Fuerzas Armadas ESPE, Universidad Técnica de Ambato, Escuela Superior Politécnica de Chimborazo, Universidad Nacional de Chimborazo, and Grupo de Investigación ARSI, for the support to develop this work.

\section{References}

[1] Chiluisa Max.G, Mullo R.D and Andaluz V.H. (2018). Training in Virtual Environments for Hybrid Power Plant. Advances in Visual Computing, 193-204.

[2] Andaluz V.H, Castillo-Carrión D, Miranda R.J, and Alulema J.C. (2017). Virtual Reality Applied to Industrial Processes. Springer International Publishing AG, 59-74. 
[3] García-Peñalvo F.J, Cruz-Benito J, Griffiths D, and Achilleos A. (2015). Tecnología al Servicio de un Proceso de Gestión de Prácticas Virtuales en Empresas: Propuesta y Primeros Resultados del Semester of Code. IEEE-ES, 52-59.

[4] Chicaiza E.A, De la Cruz E.I and Andaluz V.H. (2018). Augmented Reality System for Training and Assistance in the Management of Industrial Equipment and Instruments. Springer Nature Switzerland AG, 675-686.

[5] Zovko M. E., Dillon J. (2017). Humanism vs. competency: Traditional and contemporary models of education. Educational Philosophy and Theory, 50, 554-564.

[6] Yu Y, Duan M, Sun CH, Zhong Z, and Liu H. (2017). A virtual reality simulation for coordination and interaction based on dynamics calculation. Taylor \& Francis Group, 12, 873-884.

[7] Gonzaga L., Veronez M., Kannenberg G., Alves D., Cazarin C., Santana L., Fraga J., Leonardo C.., Souza L., Marson F., Bordin F., and Francisco M. (2018). Immersive Virtual Fieldwork: Advances for the Petroleum Industry. IEEE COMPUTER SOCIETY, 561-562.

[8] Hutton C., and Suma E. (2016). A realistic walking model for enhancing redirection in virtua reality. IEEE Virtual Reality (VR), 183-184.

[9] Wang Xu, Di Wei and Cheng Lei. (2016). Control for the Centrifugal Pump in the Simulation Platform of Power Plants. Springer Nature Switzerland AG, 264-267.

[10] Koltun G, Kolter M and Vogel-Heuse B. (2018). Automated Generation of Modular PLC Contro Software from P\&ID Diagrams in Process Industr. IEEE Institute of Automation and Information Systems, Technical University of Munich, Munich, Germany, 978-985.

[11] Andaluz V.H, Amaquiña J.L, Quevedo W.X, Mora-Aguilar J, Castillo-Carrión D, Miranda R.J and Pérez M.G. (2018). Oil Processes VR Training. Springer Nature Switzerland AG, 712-724

[12] Arroyo E, Hoernicke M, Rodríguez P, and Fay A. (2016). Automatic derivation of qualitative plant simulation models from legacy piping and instrumentation diagrams. Computers \& Chemical Engineering, 112-132.

[13] Hahn A, Hensel S, Hoernicke M, and Urbas L. (2017). Concept for the detection of virtua functional modules in existing plant topologies. IEEE 14th International Conference on Industria Informatics, 820-825.

[14] Toghraei M. (2019). Principles of P\&ID development: the tips provided here will streamline efforts to develop piping \& instrumentation diagrams. Chemical Engineering. , 62.

[15] Saguarduy J. (2016) . MathWorks. Available:https://la.mathworks.com/matlabcentral/ fileexchange/56568-pump-speed-control-water-hammer-pressure-waves.

[16] INEN. (2013). Simbolos gráficos. Colores de seguridad y señales de seguridad. Available: https://www.aguaquito.gob.ec/wp-content/uploads/2018/01/IN-3-NORMA-TECNICANTNINEN-ISO-3864-12013-S\%C3\%8DMBOLOS-GR\%C3\%81FICOS-COLORESDESEGURIDAD-Y-SE\%C3\%91ALES-DE-SEGURIDAD.pdf.

[17] NORMALIZACION, I. E. (05 de 2013). INEN. Recuperado el 12 de 02 de 2019, Available https://www.ecp.ec/wp-content/uploads/2017/10/INEN_ISO_3864 